\title{
Closed Loop Force Control of In-Situ Machining Robots using Audible Sound Features
}

\author{
David Alatorre, Amir Rabani, Dragos Axinte*, David T Branson III
}

\begin{abstract}
Detecting, measuring and controlling the forces between cutting tools and machined components is essential in circumstances where direct position control (e.g. depth of cut, feed speed, etc.) is inaccurate and/or impossible. This paper explores the use of airborne sound signals that result from the machining process to control the cutting force in closed loop for generating accurate machined features when performing in-situ robotic repair of complex installations. The sound signals during indentation at various cutting forces are analysed and used to calibrate a remotely mounted microphone sensor and signal processing control system. The power spectral density of the audible sound is used to estimate tool cutting force and the sound intensity used in turn to estimate the resulting process energy. The described controller uses intensity of sound to mitigate the effects of resonance with workpiece natural frequencies while controlling the spindle velocity of the tool based on the dominant audible frequency. The performance of the controller is validated using a representative test rig and demonstrated using a robotic arm to machine thin Ni-Cr-Co alloy cantilever beams with a miniature air-driven grinding tool. Results from the test rig show that such a sound-based control approach can achieve consistent cutting forces with an accuracy of $0.08 \mathrm{~N}$. The robot arm is shown to be capable of grinding features of consistent depth (to within $0.05 \mathrm{~mm}$ ) on beams with surface defects of undefined shape using only the sound of the process for closed loop force control.
\end{abstract}

Key words: Force control, Machining, Aircraft Maintenance, Airborne Sound, Power Spectral Density, Sound Intensity.

\section{Introduction}

Repair of high-value industrial installations is often carried out in-situ due to the costs associated with the disassembly and the need to transport the parts to repair workshops. However, the main difficulty when performing in-situ repairs of components or assemblies is often the accessibility into crammed spaces which sometimes can only be reached through narrow and winding passages, as presented in Figure 1 for a gas turbine engine. The intervention in tight workspaces also means that expert knowledge is necessary to carry out maintenance work without damaging surrounding objects.

Traditionally, whenever possible, in-situ repair work on industrial installations such as gas turbine engines has been performed manually by use of specialist tooling with cutting, grinding and polishing end effectors to name a few. Due to this operational complexity there is growing interest in automating these repair interventions by using robotic instruments. In such instances, chellenges in accessibility result in maintenance instruments that are long and slender $[1,2]$ which in turn limit the possibility to embed sensing systems (e.g. force or tactile) to support accurate reach of the target workspace. Thus, to ensure quality of the in-situ interventions, the probes must be equipped with miniaturised sensing

\footnotetext{
${ }^{*}$ Corresponding author. Tel.: +44 1159514117 E-mail: Dragos.Axinte@nottingham.ac.uk Present address: Advanced Manufacturing Building, University of Nottingham, Nottingham NG8 1BB, UK
} 


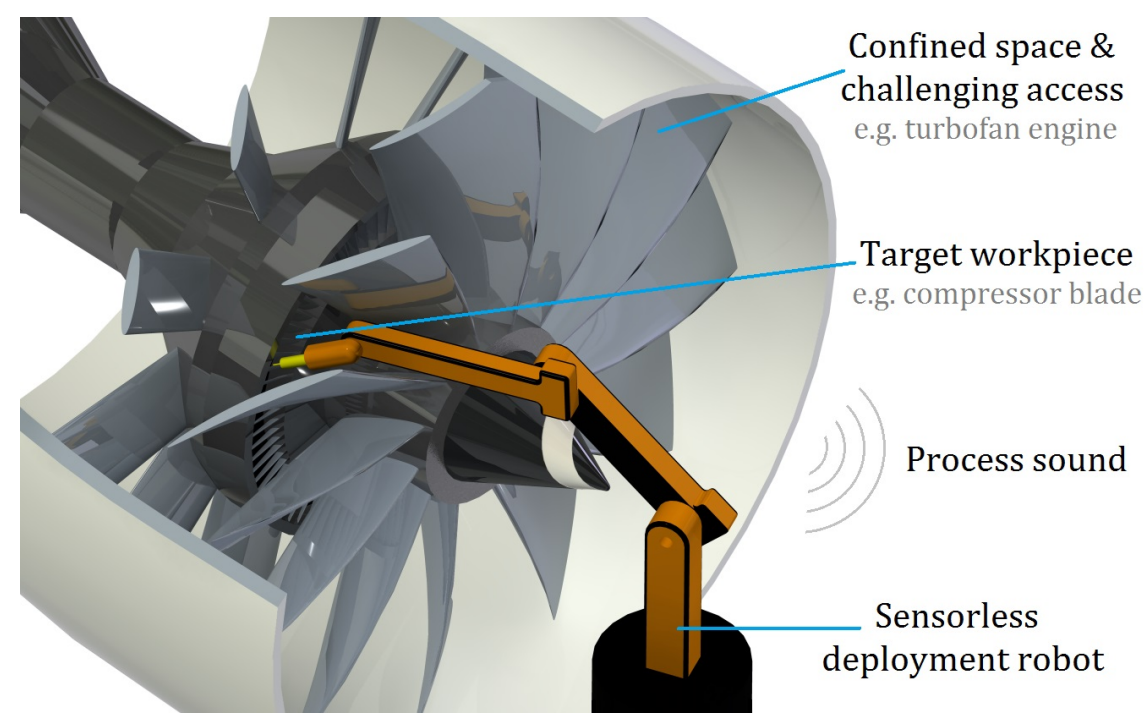

Figure 1: An example of in-situ repair of high-value equipment using robots with limited sensing capabilities to access difficult-to-reach components.

systems, or assisted by remote sensors placed some distance away and using propagated signals (e.g. acoustic emission, light, sound) to optimise and control the in-situ intervention.

This work explores the use of high-speed, low-torque, air-driven grinding tools for material removal in confined spaces. The nature of the tool results in low contact forces and low material removal rates. For closed-loop force control to be achieved, the feedback method must be sensitive enough for this application.

\section{$1.1 \quad$ Background}

Current methods of closed loop control using force signals for in-situ instruments are generally reliant on direct feedback from sensors (e.g. load cells, strain gauges). For example in [3], load cells mounted on the cables at the proximal end of a continuum robot were used to measure forces acting on the distal end. Other cable-driven manipulators such as those used for minimally invasive surgery have also been fitted with similar sensors for force measurement and control [4-6]. However, these methods rely on accurate knowledge of the instantaneous configuration and geometry of the instrument, which is a difficult task considering the possible deflection of continuum robots. In addition to the limitations of force sensing via tendons on long robots, the approach cannot be used on a sensorless robot without modifying it by adding load cells.

An alternative method could be to use Acoustic Emission (AE), due to its high signal-to-noise ratio, to monitor machining operations. In [7], AE was used to monitor surface quality during machining of nickel-based superalloys. In [8], AE was used to detect dullness of grinding wheels. Other authors have also demonstrated the use of AE to monitor different machining parameters [9-13]. Similarly, vibration signals measured with an accelerometer and amplified with a vibrometer were used to monitor parameters during grinding in [14].However, despite its numerous advantages, the AE sensor or accelerometer needs to be mounted directly on the surface where the waves are to be captured, which is impossible when remote workspaces are accessed for performing in-situ material removal interventions.

Audible sound features have also been used to monitor or predict various parameters of machining processes and machine tools performance. In [15], airborne sound was used to identify appropriate abrasive densities during honing. Rubio et al. [16] used frequency signatures of audible sound to identify cutting parameters during milling, such as rotation speed and depth of cut. Other authors have used sound signal-processing methods - RMS of pressure, intensity and frequency spectrum 
of the signals - to monitor or predict different quality parameters (e.g. surface roughness, chatter) during band sawing $[17,18]$. However, no attempt is made to control the machining parameters in closed loop using the airborne sound features.

Robben et al. [19] described a method for determining tool settings and predicting the material composition of concrete (i.e. rock and cement content) during disk cut-off grinding with diamond wheels using airborne sound features, and were able to create a map of the hardness and compositions of the concrete slabs. Khan et al. [20] used sound features (such as RMS of microphone voltage and cumulative amplitudes of dominant frequencies) to determine wear damage in mechanical components with multiple contact points under load. These reports show extensive efforts on the use of features of sound signals for process monitoring but, it seems, no examples of employing sound signals to control the material removal processes in closed loop have been reported.

While airborne sound signals are more susceptible to distortion and noise, the sensors can be placed some distance away from the machining operation making them more suitable for the in-situ repair application. The objective of this work is to identify a method for closed loop force control that is applicable to sensorless robots and useful for repair of difficult-to-access industrial assets.

In this context, a robotic tool equipped with a method for online process monitoring using sound signals derived from the machining operation can be highly efficient for closed loop control of different operations (e.g. grinding, milling) in inaccessible and especially in blind workspaces. With such facilities in place, automated in-situ repair applications could be performed with precision and reliability.

\subsection{Contributions}

This paper describes a novel method for using audible sound features to control cutting forces during grinding in closed loop to assist automated in-situ robotic repair of complex industrial systems. A methodology to obtain the relation between dominant sound frequencies, sound intensity and cutting forces is described. To measure the sound output of the grinding process, a representative test rig with the necessary sound, force and cutting speed sensors has been set up. A novel controller is presented, which uses sound intensity measurements to mitigate the effects of resonance with workpiece natural frequencies. The controller uses dominant audible frequency measurements to control the spindle velocity and hence the forces between tool and workpiece. In this way, actuators with no intrinsic force sensing capabilities can be operated with closed loop force control. This is achieved for an actuated arm with a grinding end effector and demonstrations are carried out through automated grinding of near-constant-depth features on metallic components with unknown topology.

Section 2 of the paper discusses the characterisation and experimental validation of the sound features associated with cutting force in grinding and introduces the methods used for signal processing. Section 3 describes the procedure used to calibrate and test the acoustic sensor for closed loop control. Section 4 discusses the outcome of the machining operations using closed loop control and the possible applications in wider industries.

\section{Characterisation of Sound Features for Force Control}

The predominant audible sound features that arise from a machining operation such as grinding can be predicted based on an understanding of the core phenomena. A method is described here to obtain and analyse the sound features and demonstrate that the sound emissions of a given system can be used for closed loop force control of a robot for in-situ grinding within remote spaces and without the need for additional sensing. 


\subsection{Description of audible sound emissions during grinding}

The airborne sound generated during grinding using miniature air-driven tools is expected to come primarily from the following sources:

1. The sounds caused by periodic impacts between the abrasive elements or grits of the tool and the workpiece. The frequency of these contacts is dictated by the size and spatial distribution of the abrasive grains, the diameter, and the speed of rotation of the tool $S$. Common grinding tools operate at over 20sm/s (surface metres per second) [21] and have small $(<1 \mathrm{~mm})$ grains that are randomly distributed resulting in ultrasonic white noise. Wear of the grinding bur influences the distribution of grits and affects this sound feature.

2. The sounds associated with the rotating turbine of the air-driven spindle and grinding tool. Cyclical events related to whole rotations of the tool such air passing turbine blades and oscillations of the grinding tool contribute to sound with a frequency that matches the speed of rotation. This can be verified using non-contact instruments such as laser tachometers.

3. The natural frequencies of the workpiece. The mechanical action of the grinding tool could excite the workpiece at its natural frequencies and it is possible to predict the resulting sound emissions using finite element analysis. This information can be supported with measurements acquired through impact testing and laser vibrometry equipments. Higher cutting forces result in more energy being transferred to the workpiece in the form of vibration.

4. The noise generated by the exhaust air from the air-driven spindle. The sound produced by the venting air is determined by the diameter and pressure drop across the exhaust holes. The range of noise frequencies produced by this exhaust air depends on the speed of rotation of the turbine [22].

5. The noise arising from echoes and interference with the surroundings. Complex industrial installations (such as gas turbine engines) present geometries that can reflect and distort sound, and thus could affect the power and direction of the signals. However, due to the high geometrical variety of workspaces where the robotic system for in-situ grinding can operate, interference effect are only be discussed in relation to the observations during closed loop testing.

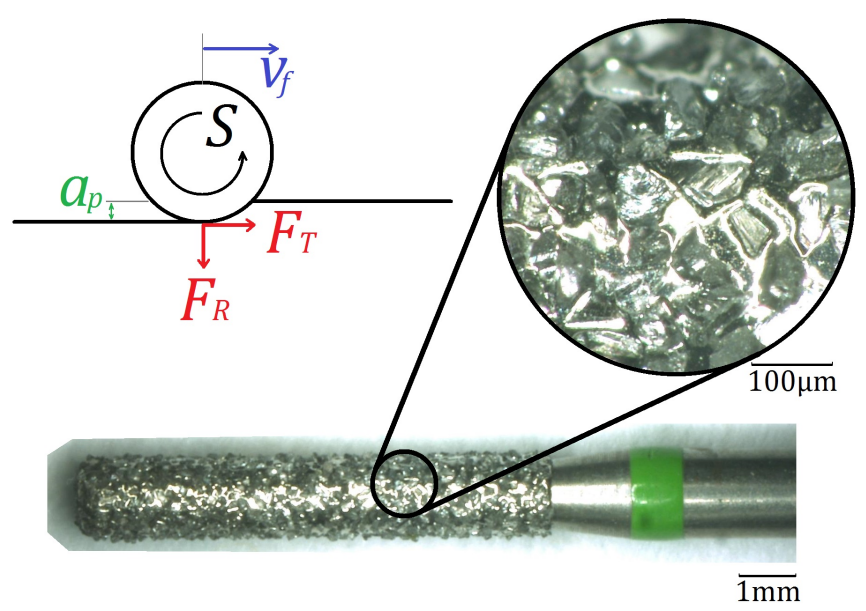

Figure 2: Representation of a small grinding tool driven by a miniature air-driven spindle and the forces, velocities and displacements of the tool relative to workpiece surface.

It is documented [23] that air-driven high-speed spindles (such as those used in this work) have nearlinear torque-speed relations. When employed with grinding tools, an increase in the forces associated 
with chip formation, ploughing of material, and friction between tool and workpiece [24] leads to a decrease in spindle velocity. Although the tangential and radial forces $\left(F_{T}\right.$ and $F_{R}$ in Figure 2) also depend on factors such as workpiece material, feed speed, grit size, type of abrasive grains, etc. [21], the behaviour of a particular air spindle, tool and workpiece combination can be obtained experimentally. Hence, by identifying, tracking and controlling the sound frequency associated with spindle rotation speed (item two on the list above), it is possible to control grinding force in closed loop.

A theoretical torque/speed line can be obtained from the stalling torque $\tau_{s}$ and the idle spindle velocity $S_{i d l e}$ of the turbine tool using Equation 1.

$$
\tau=\tau_{s}-\frac{\tau_{s} * S}{S_{i d l e}}
$$

The ratio of tangential force to radial force, $\mu$, changes depending on the level of engagement of the abrasive tool with the workpiece material (Equation 2). An increase in engagement coefficient $\mu$ leads to an increase of the tangential force observed for a given radial force, shown in Figure 3 as a change in $F_{R} / S$. This engagement coefficient can change as a result of increasing feed speed $v_{f}$ at a given radial force $F_{R}$. Furthermore, tool wear and the resulting reduction in grit efficacy can also change the engagement coefficient, although quantifying this change is outside the scope of this work.

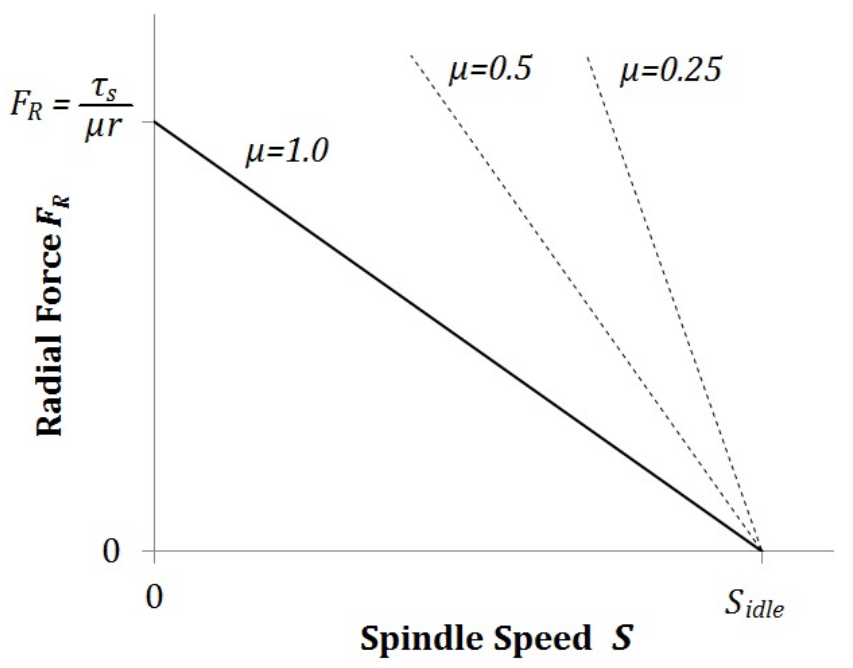

Figure 3: Theoretical dependence radial force v. spindle speed for an air turbine tool of radius $r$ with engagement coefficient $\mu$.

$$
\mu=F_{T} / F_{R}
$$

Given that the spindle speed can be measured by analysing the dominant sound frequencies (those associated with cyclical events), knowledge of the real torque-speed line can be used to indirectly estimate the radial force relative to a reference value and hence to enable closed loop force control of the grinding process.

\subsection{Acquisition of calibration data}

Before implementing this approach on a robotic system for automatic grinding, a calibration test rig consisting of a grinding tool mounted on a pivoting arm with variable offset mass (Figure 4) was designed to obtain the sound signals associated with different levels of radial force $F_{R}$. The offset mass ranges from $0 \mathrm{~g}$ to $135 \mathrm{~g}$ with $45 \mathrm{~g}$ increments, giving a range of radial contact forces of 0 to $1.32 \mathrm{~N}$. The mass is isolated from the vibrations of the machining process by a spring-damper. An adjustable 


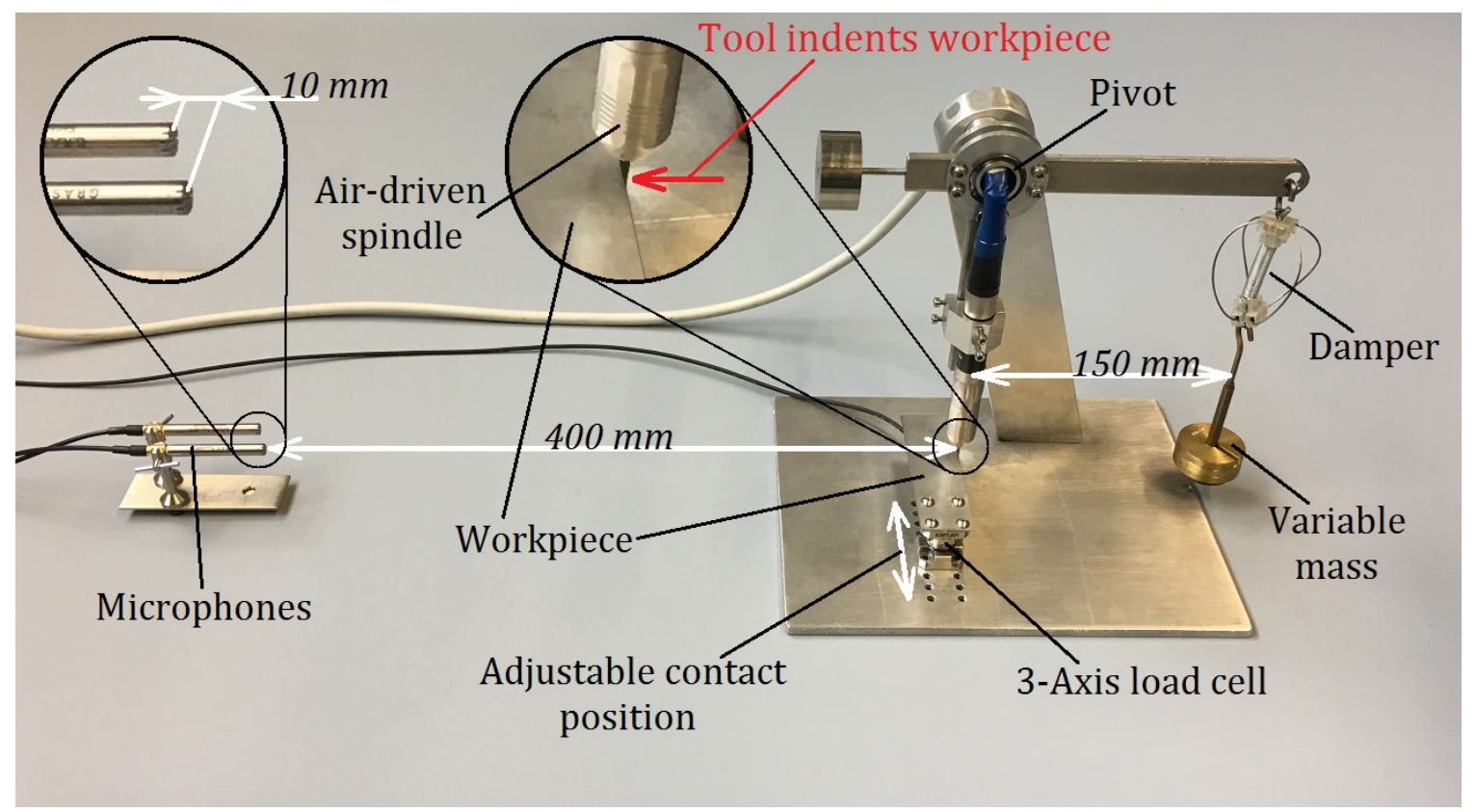

Figure 4: Test rig for monitoring audible sound signals during grinding at different radial force intervals. Variable offset mass was used to control the radial force.

counterweight is used to level the tool for a radial force close to $0 \mathrm{~N}$ when unloaded. When the air spindle is operating, the offset mass forces the grinding tool into the workpice creating an indentation. In addition to the radial cutting force, the position of the contact along the length of the workpiece can also be adjusted and was randomised during the tests to nullify any effect of location of contact on the results.

A miniature air-driven spindle (Presto Aqua Lux NSK-Nakanishi) with a maximum idle (unloaded) speed of $350 \mathrm{kRPM}(S=5833 \mathrm{~Hz})$ and a stalling torque of $\tau_{s}=1.22 \mathrm{mNm}$ [23] was used to grind workpieces $(100 \mathrm{~mm} \times 30 \mathrm{~mm} \times 1.6 \mathrm{~mm})$ made of Nickel-Chromium-Cobalt alloy. The workpiece was attached from one end as a cantilever such that the natural frequencies of the beam could be calculated with ease. A new $1.6 \mathrm{~mm}$ diameter $118 \mu \mathrm{m}$ grit electroplated diamond grinding tool (Edenta AG) was fitted to the spindle for each of the tests to nullify any effect of tool wear on the results.

The workpieces were mounted on a 3-axis dynamometer (9317B Kistler) with a sensitivity of $25.71 \mathrm{pC} / \mathrm{N}$ in $X$ an $25.48 \mathrm{pC} / \mathrm{N}$ in $Y$ to measure the cutting forces $F_{R}$ and $F_{T}$ respectively during calibration grinding tests. The dynamometer was used with its low-range settings $( \pm 60 \mathrm{~N}$ in $X$ and $Y$ ). A Kistler multichannel charge amplifier (Type 5017), with an effective resolution for this sensor of $0.39 \mathrm{mN}$, was used throughout this work. Preliminary trials with this setup showed a background noise standard deviation of $1.95 \mathrm{mN}$ and an average signal-to-noise ratio during grinding of $32.2 \mathrm{~dB}$.

Two 4OPH CPP free-field array microphones with 12AL CPP pre-amplifiers (G.R.A.S. Sound \& Vibration), with operating frequency range between $10 \mathrm{~Hz}$ and $20 \mathrm{kHz}$, were positioned $400 \mathrm{~mm}$ from the spindle. This distance was chosen to limit free-field spherical propagation loss and remain realistic for an in-situ repair operation. Two microphones were used with a $10 \mathrm{~mm}$ gap between them to obtain the pressure gradient between two points for the intensity calculation. Sound signals were acquired for 0.1 seconds at $50 \mathrm{kHz}$ using an EMP373 portable computer (ACME) equipped with a PCI-6229 IO board (16 bit, $250 \mathrm{kS} / \mathrm{s}$ ) and a $B N C$-2110 BNC breakout board (National Instruments).

Tests were repeated eighteen times for every level of radial force in a random order as shown in Table 1.

For every trial, the air spindle was started away from the workpiece to allow it to reach idle speed and placed to rest against the workpiece with no transverse movement. Sample signals were collected 
Table 1: Description of calibration experiment trials.

\begin{tabular}{|l|c|l|}
\hline Factor & No. of Levels & Description of Levels \\
\hline Radial cutting force & 4 & $\approx 0 N$ \\
& & $0.4 N$ \\
& & $0.8 N$ \\
& & $1.2 N$ \\
\hline Position of contact & 3 & Three points along the \\
& & workpiece $30 \mathrm{~mm}$ apart \\
\hline Repetitions & 6 & \\
\hline
\end{tabular}

two seconds after the tool contact with the workpiece to ensure that any vibration of the offset mass had been attenuated by the damper.

\subsection{Acoustic sensor calibration}

After collecting sound samples from multiple grinding indentations, the following methodology was followed to extract useful sound features for force control.

\subsubsection{Dominant audible frequency}

The power spectral density (PSD) of the sound in $V^{2} / H z$ was calculated to detect the frequencies associated with the sources described in Section 2.1. It was observed that, generally, the audible frequency with the highest magnitude is associated with the rotation of the spindle (as seen in the example shown in Figure 5). Other peaks are associated with the resonant frequencies of the workpiece and the sound of exhaust air. To capture those frequencies related to spindle rotation (for closed loop control), a Butterworth highpass filter was implemented to remove frequencies lower than $500 \mathrm{~Hz}$, as they can be ignored as background noise. Force was acquired from the dynamometer in the $F_{R}$ direction to be correlated to the sound monitored spindle speed.

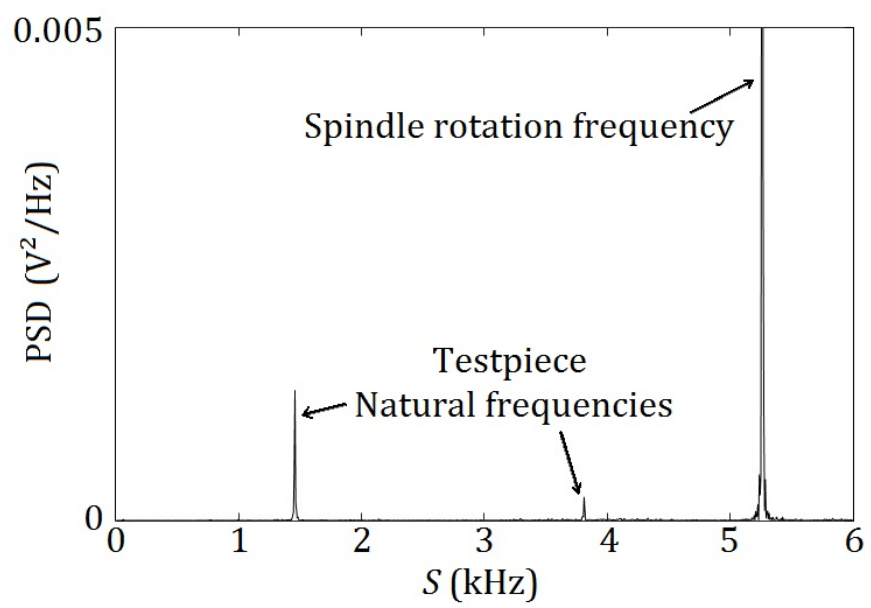

Figure 5: Power spectral density plot at audible frequency $S$ for a typical sample with electroplated diamond grinding tool.

The plot of radial force against spindle speed (Figure 6) shows that the slowing of the tool rotation 
at increased radial force can be measured using sound features (PSD peaks). The increased variance (horizontal error bars show one standard deviation) at high radial forces can be attributed to the sound arising from the natural frequencies of the vibrating workpiece overpowering the sound of the rotating spindle, making it difficult to track the spindle speed.

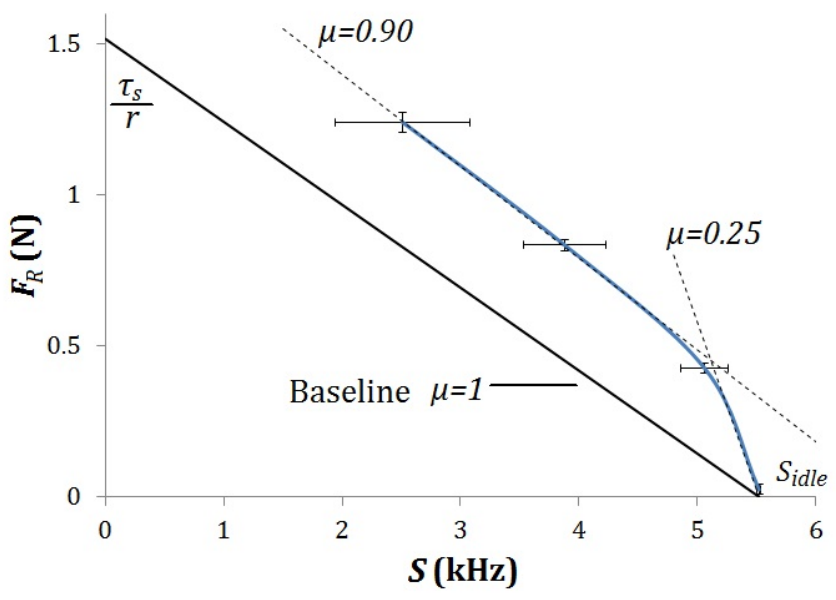

Figure 6: Measured radial force $F_{R}$ vs. spindle speed $S$ (dominant audible frequency). Dotted lines show engagement coefficient $\mu$ during sliding friction $(\mu=0.25)$ and after the tool's abrasive grains engage the workpiece $(\mu=0.90)$.

The change in engagement coefficient from $\mu=0.25$ to $\mu=0.9$ observed in Figure 6 is attributed to a substantial rise in tangential force due to an increase in depth of cut and in energy transferred to the workpiece during ploughing [21]. The measurements are also consistent with the observations made by [24].

It is expected that tool wear will influence the engagement coefficient during operation. The rate at which this happens is explored in the closed loop control section. While tool wear does have an effect on the relation between audible features and contact force, the machining task under investigation is small (approximately 10\% to $20 \%$ of the tool life) and the contact is light enough (usually less than $50 \%$ of stall forces) that electroplated diamond burs do not experience any significant abrasive wear. The effect of wear may become important and require compensation for operations requiring more prolonged or aggressive grinding; however, when addressing in-situ repairs of industrial assets priority will often be given to replacing inexpensive tools for optimal performance.

\subsubsection{Sound intensity}

Sound intensity is associated to the energy carried by acoustic waves as they cross an area of interest and it can be calculated by measuring the pressure gradient between two points along direction $x$ perpendicular to this area [25]. Hence, the sound intensity between points $a$ and $b$ along $x$ can be expressed as:

$$
I=10 \log _{10}\left(\frac{\frac{P_{a}+P_{b}}{2 \rho \Delta x} \int\left(P_{a}-P_{b}\right) d t}{I_{0}}\right)
$$

where $\Delta x$ is the distance between points $a$ and $b, P_{a}$ and $P_{b}$ are the pressure levels at $a$ and $b$ respectively and $\rho$ is the air density. The conventional reference intensity value of $I_{0}=1 \mathrm{pW} / \mathrm{m}^{2}$ is used in this study for intensity calculations [25]. The distance between the two microphones $\Delta x$ was set to $10 \mathrm{~mm}$, as it must be smaller than the shortest wavelength expected (approx. $17 \mathrm{~mm}$ ).

The overall sound intensity level was found to increase with radial force at a rate of approx. $6.5 \mathrm{~dB} / \mathrm{N}$, as shown in Figure 7 where each marker represents eighteen data points. This rise in sound 


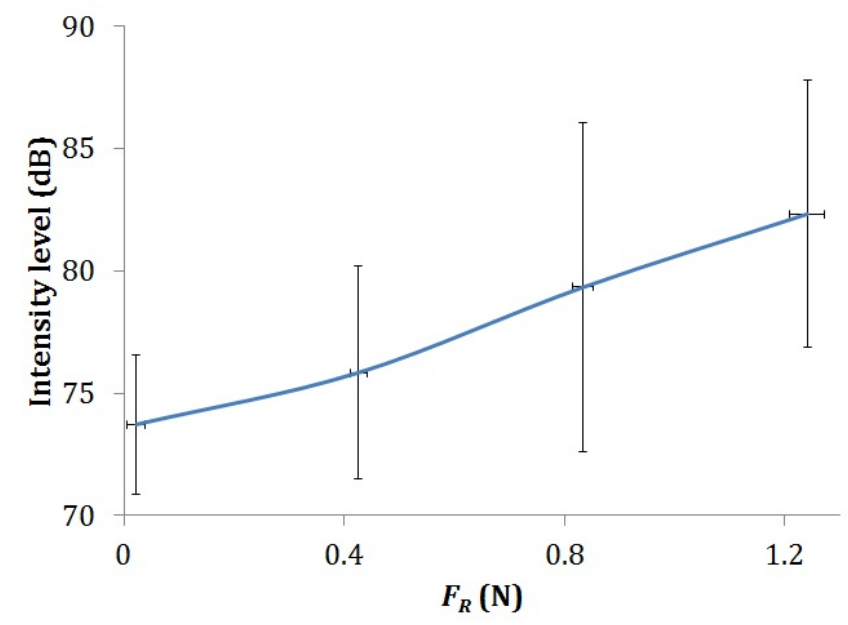

Figure 7: Sound intensity level $I$ vs. measured radial force $F_{R}$. While sound intensity does increase with force, variance between tests is high.

intensity was attributed to an increase in energy transferred to vibration of the workpiece at higher grinding forces, as the power of the natural frequencies increased together with the intensity. Although the baseline intensity varied between trials it was observed that when the grinding tool decelerated enough to match a workpiece natural frequency, the measured sound intensity increased by 5 to $10 \mathrm{~dB}$. This can be seen to some extent in the variance of the measurements shown in Figure 7, particularly at $0.82 \mathrm{~N}$ where the tool approached a resonant frequency of $3.65 \mathrm{kHz}$. Hence, despite being too variable for closed loop force control, intensity measurement can be used to identify and quantify workpiece resonance.

The dominant sound frequency can therefore be used to predict radial force, while the sound intensity can be used to predict the situation that workpiece natural frequency vibrations may overpower the sound of the spindle rotating. Together, these sound features can be used as robust feedback for a novel closed loop force controller as discussed in the following section.

\section{Closed Loop Force Control using Sound Features}

Early trials using only dominant frequency (spindle speed) as a controller input resulted in inevitable loss of control at moderate forces due to more energetic workpiece vibration frequencies overpowering the spindle velocity. The outcome varied randomly between driving the spindle into the workpiece (causing a stall) or retracting the spindle fully away from the workpiece. Subsequent improvements led to the control strategy outlined in this section.

To use sound features for closed loop force control, the controller must be calibrated using ambient noise and the sound of the spindle running at idle (unloaded) speed. The controller can then be tuned to achieve the desired behaviour.

\subsection{Controller calibration methodology}

To calibrate the controller a measurement of sound with the spindle running unloaded was taken to identify the dominant frequency (idle spindle speed) from the PSD. This idle spindle speed is used as a reference value for the case $F_{R}=0 \mathrm{~N}$. The data was then analysed to obtain the idle sound intensity using Equation 3. The idle intensity gives an indication of the total energy coming from the grinding process as well as the distance between the microphones and the source. Small variations in this distance during operation do not affect the performance of the controller after calibration, as 


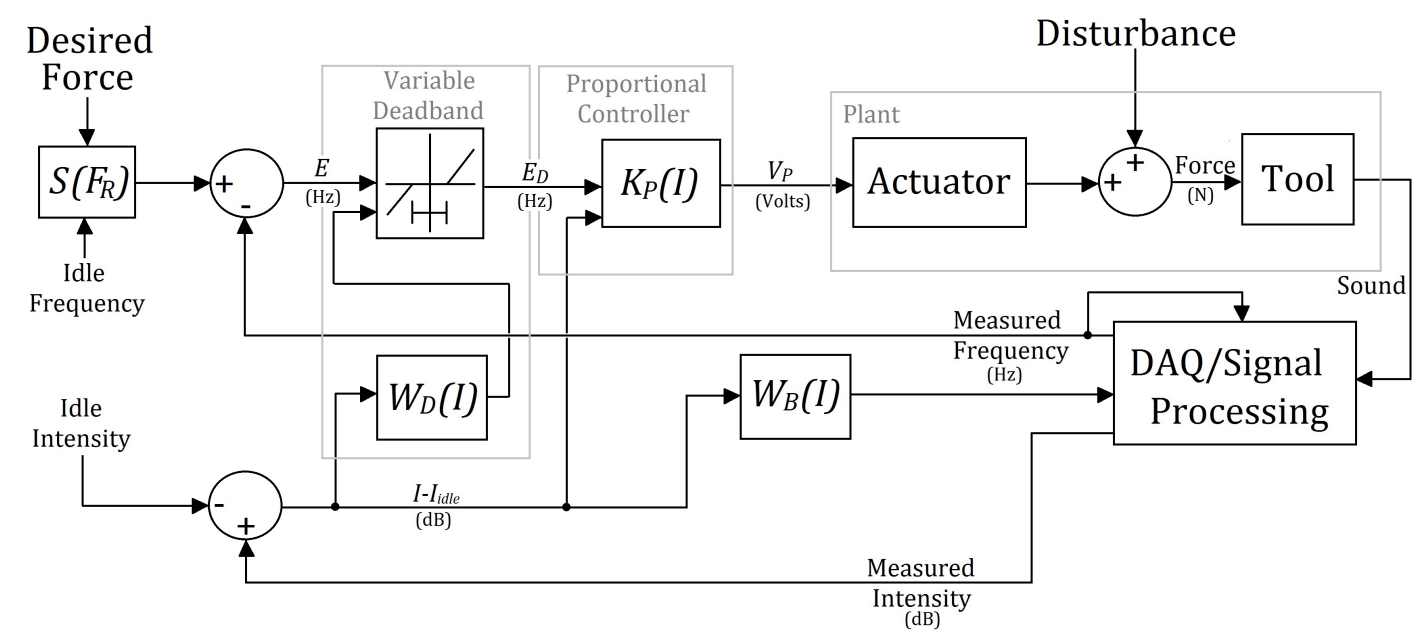

Figure 8: Proposed closed loop control of radial grinding force with single actuator using audible sound features as feedback. $W_{D}(I), K_{P}(I)$ and $W_{B}(I)$ determine the effect of intensity on deadband width, proportional gain and bandpass filter width respectively.

the intensity is expected to fluctuate naturally. However, if the distance between the workpiece and the microphones is expected to change significantly during operation (such as when machining a large feature), the propagation intensity losses associated with distance must be taken into account. In the work undertaken here, the small variations in machining distance during operation do not change intensity enough to affect the controller.

A bandpass filter centred around the highest peak of the PSD of the signal is then implemented. The value of frequency at the centre of the filter varies on each iteration of the controller, such that when applied to subsequent measurements, the filter tracks the frequency associated with spindle speed $S$ as it slows down or speeds up, provided that the changes are gradual enough to remain within the filter bandwidth.

\subsection{Closed loop controller design}

The proposed control architecture is a proportional controller with variable deadband width and variable gain (Figure 8). The controller inputs are the target force and the calibration constants for idle spindle speed and idle sound intensity. The difference between the desired spindle speed (the setpoint) and the measured $S$ is multiplied by a proportional gain to obtain a value to drive the plant actuator (e.g. an analogue voltage signal). The actuator affects the radial force between the air-driven tool and the workpiece, changing the sound of the grinding process. The data acquisition and control equipment captures and processes the signals from the microphones to obtain values of frequency and intensity for the following control loop. The microphones response frequency is $20 \mathrm{kHz}$, and sound is sampled at $50 \mathrm{kHz}$. Due to the length of the captured signal on each iteration $(0.1 s$ to allow enough data for PSD and filters robustness), the effective frequency of the control loop is $10 \mathrm{~Hz}$. Hence, the response of the force controller to perturbations is attenuated at frequencies higher than approximately $5 \mathrm{~Hz}$.

\subsubsection{Target Frequency}

It was assumed that the engagement coefficients during light contact $\left(\mu_{1}=0.25\right)$ and grinding $\left(\mu_{2}=\right.$ 0.9) remain constant (negligible tool wear and feed speed) regardless of the idle velocity of the tool. In circumstances where tool wear is significant $\mu_{2}$ can be defined as a variable that changes with time, ground distance, material removed, etc. according to further calibration experiments. The gradual 
transition between the two slopes occurs at radial force $F_{\text {eng }}$ and can be approximated using a smooth interpolation function of the form

$$
\frac{1+\tanh \left(k\left(F_{R}-F_{\text {eng }}\right)\right)}{2}
$$

where $k \in \mathbb{R}$ and determines how gradual or abrupt the transition is.

Interpolating the two engagement coefficient lines gives an equation of best fit (5) that can be used to identify the target spindle speed $S$ based on a desired radial force $F_{R}$, and to be used as a setpoint during closed loop control (Figure 8).

$$
S=S_{\text {idle }}\left(\left(1-\frac{r}{\tau_{s}} F_{R} \mu_{1}\right)+\frac{1+\tanh \left(k\left(F_{R}-F_{\text {eng }}\right)\right)}{2}\left(\frac{r}{\tau_{s}}\left(F_{R}\left(\mu_{1}-\mu_{2}\right)+F_{\text {eng }}\left(\mu_{2}-\mu 1\right)\right)\right)\right)
$$

A value of 4 was chosen for $k$ to achieve the smooth transition seen in Figure 6 .

\subsubsection{Variable Deadband}

A deadband with variable width is introduced to make the controller capable of ignoring variations in frequency resulting from small disturbances in force during machining without excessively lowering the proportional gain. Here, an increase in sound intensity indicates that the workpiece is vibrating with more energy at its natural frequencies. If the spindle velocity approaches an overpowered natural frequency, the controller will mistake the natural frequency for a change in tool speed and take undesired corrective action. The effect of this natural frequency can be minimised by making the width of the deadband $W_{D}$ a function of sound intensity $I$ and idle intensity $I_{i d l e}$. Therefore, the input error to the proportional controller (see Figure 8) can be expressed as:

$$
E_{D}= \begin{cases}E+\frac{W_{D}(I)}{2} & \text { for } E<-\frac{W_{D}(I)}{2} \\ 0 & \text { for }-\frac{W_{D}(I)}{2} \leq E \leq \frac{W_{D}(I)}{2} \\ E-\frac{W_{D}(I)}{2} & \text { for } \frac{W_{D}(I)}{2}<E\end{cases}
$$

where $E$ is the difference between the setpoint frequency and the measured frequency; $E_{D}$ is the error used for proportional control. The deadband width $W_{D}(I)$ has upper and lower saturation limits $\sigma_{H D}$ and $\sigma_{L D}$ respectively and is given by:

$$
W_{D}(I)=\operatorname{sat}\left[\left(I-I_{i d l e}\right) * K_{D}\right]_{\sigma_{L D}}^{\sigma_{H D}}
$$

where $\operatorname{sat}[x]_{L}^{H}=\max (L, \min (H, x))$. The values of deadband gain $K_{D}$ and other constants are shown in Table 2.

Table 2: Control constants used in closed loop controller tests.

\begin{tabular}{|ll|ll|ll|}
\hline$K_{D}$ & 35 & $K_{P}$ & 0.14 & $K_{B}$ & 45 \\
$\sigma_{H D}$ & 150 & $\sigma_{H P}$ & 0.6 & $\sigma_{H B}$ & 250 \\
$\sigma_{L D}$ & 75 & $\sigma_{L P}$ & 0.1 & $\sigma_{L B}$ & 100 \\
\hline
\end{tabular}

\subsubsection{Proportional Controller}

Similar to the deadband, the proportional gain constant $K_{P}$ is tuned to lower the proportional gain of the controller when the intensity of sound exceeds a given threshold above idle intensity, preventing 
large inputs by the actuator when faced with resonant interference. Hence, the effective control signal or voltage from the proportional controller $V_{P}$ is given by:

$$
V_{P}=E_{D} * \operatorname{sat}\left[\left(\sigma_{H P}+\sigma_{L P}\right)-\left(I-I_{i d l e}\right) * K_{P}\right]_{\sigma_{L P}}^{\sigma_{H P}}
$$

where $\sigma_{H P}$ and $\sigma_{L P}$ are the upper and lower bounds of the proportional gain. The ouputs of the controller are a direction signal and pulse width modulation signal to the actuator H-bridge. A proportional controller was chosen over a PI, PD or PID controller due to the relatively high latency caused by the acquisition of sound signals. The introduction of integral or derivative terms was not found to improve stability in this case (see future work).

\subsubsection{Plant}

The output voltage/signal from the control hardware drives an actuator (shown in Figure 9) that moves the tool towards or away from the workpiece, changing the radial force acting on the tool and hence the depth of cut. The plant is the physical system and can be described as a number of processes in series including backlash, actuator response time, robot kinematics, tool torque/speed characteristics and airborne signal time delays and losses (Figure 8).

\subsubsection{Data Acquisition and Signal Processing}

Every 0.1 seconds, the signal from the two microphones (sampled at $50 \mathrm{kHz}$ ) is analysed to obtain the intensity of sound coming from the workpiece (Equation 3). A Butterworth bandpass filter centred around the dominant frequency calculated on the previous measurement $S_{z-1}$ ignores any sound that is not related to the spindle rotation, and provided this speed has not changed dramatically since the previous measurement, the highest PSD of the filtered signal gives the dominant frequency of the current measurement $S_{z}$. Like the variable deadband and proportional controller, a bandwidth function, $W_{B}(I)$, controls the width of the bandpass filter used to isolate the spindle speed frequency from background noise. When the energy of the workpiece vibration rises due to resonance or changes in background noise, the bandwidth narrows to further isolate the signal from the noise. The bandwidth function, $W_{B}(I)$, can be defined as:

$$
W_{B}=\operatorname{sat}\left[\left(\sigma_{H B}+\sigma_{L B}\right)-\left(I-I_{\text {idle }}\right) * K_{B}\right]_{\sigma_{L B}}^{\sigma_{H B}}
$$

where $\sigma_{H B}$ and $\sigma_{L B}$ are the upper and lower limits of the filter width, the values of which are shown in Table 2.

In summary, the microphones listen to the process and a bandpass filter isolates the frequency associated with the rotation of the spindle. On each control cycle, the bandpass filter moves to centre around the highest power frequency, effectively tracking the spindle speed. As the tool slows down and excites workpiece natural frequencies, sound intensity increases and the controller reacts by preemptively increasing the deadband, lowering the proportional gain and narrowing the bandwidth of the filter, reducing the risk of a natural frequency being mistaken for the spindle rotation. The spindle velocity is controlled in closed loop to achieve the desired radial force.

\subsection{Experimental validation of the controller}

The controller design described in Section 3.2 was implemented on LabVIEW software at a rate of $10 \mathrm{~Hz}$ using the control constants outlined in Table 2. The EMP373 portable computer equipped with a PCI-6229 IO board was used to gather data and send control signals to the actuators. The operation of the controller was verified using a test rig (Figure 4) and demonstrated using a robotic arm in an environment resembling a complex industrial enclosure. 


\subsubsection{Controller performance tests}

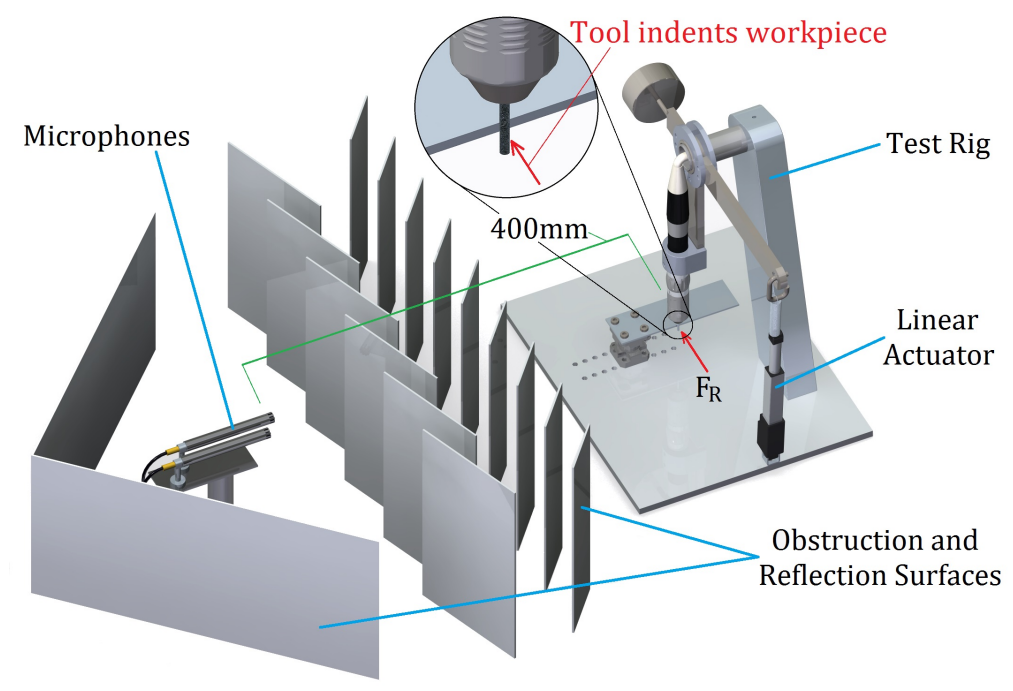

Figure 9: Test setup for controller validation. Microphones are separated from the source of the sound by a number of obstruction and reflection surfaces during some tests.

The performance of the controller was initially validated using the cantilever test rig to measure the radial force and material removal rate of the grinding tool with and without closed loop force control. For each trial the tool contacted the workpiece for 60 seconds, the contact forces were measured with the $9317 B$ dynamometer and the volume of removed material was calculated by measuring the size of the indentation using a VHX-100 digital microscope (Keyence Corporation). The trials at constant radial force were carried out using the offset mass test rig (Figure 4) at six radial force intervals from 0.22 to $1.32 \mathrm{~N}$ (increments of $0.22 \mathrm{~N}$ ). The offset mass was then replaced with a $212-50-100-12-P$ DC geared linear actuator (Actuonix Motion Devices) for the tests with closed loop force control (Figure 9) at four radial force targets from 0.3 to $1.2 \mathrm{~N}$ (increments of $0.3 \mathrm{~N}$ ). When the air spindle is operating, the linear actuator forces the grinding tool into the workpice creating an indentation. The closed loop force control trials were conducted first with direct line of sight (no obstructions) between the microphone and the workpiece. Finally, the same experiments were completed with reflection and obstruction surfaces resembling the stator and rotor blades of an aeroengine shown in Figure 9, to determine whether such obstacles affect the controller. All tests were repeated nine times.

Table 3: Standard deviation values for obstructed and unobstructed closed loop control tests.

\begin{tabular}{|c|c|c|}
\hline $\begin{array}{c}\text { Setpoint } \\
(\mathrm{N})\end{array}$ & $\begin{array}{c}\text { Unobstructed } \\
\text { st. dev. }\end{array}$ & $\begin{array}{c}\text { Obstructed } \\
\text { st. dev. }\end{array}$ \\
\hline 0.3 & 0.029 & 0.030 \\
0.6 & 0.041 & 0.044 \\
0.9 & 0.045 & 0.045 \\
1.2 & 0.077 & 0.066 \\
\hline
\end{tabular}

Figure 10 shows the radial force deviations observed during closed-loop control at each target force. Deviations were calculated by comparing the mean force during each trial to the desired force. Bars on the plot shows the standard deviation for each setpoint, also shown in Table 3. The results show that the controller achieves effective tracking, with the worst performance characterised by a precision (standard deviation) of $0.08 \mathrm{~N}$ (unobstructed $1.2 \mathrm{~N}$ ) and a trueness (average mean error) of $0.02 \mathrm{~N}$ (unobstructed $0.9 N$ ). Hence, the sensor is said to have an accuracy of $0.08 N$. 

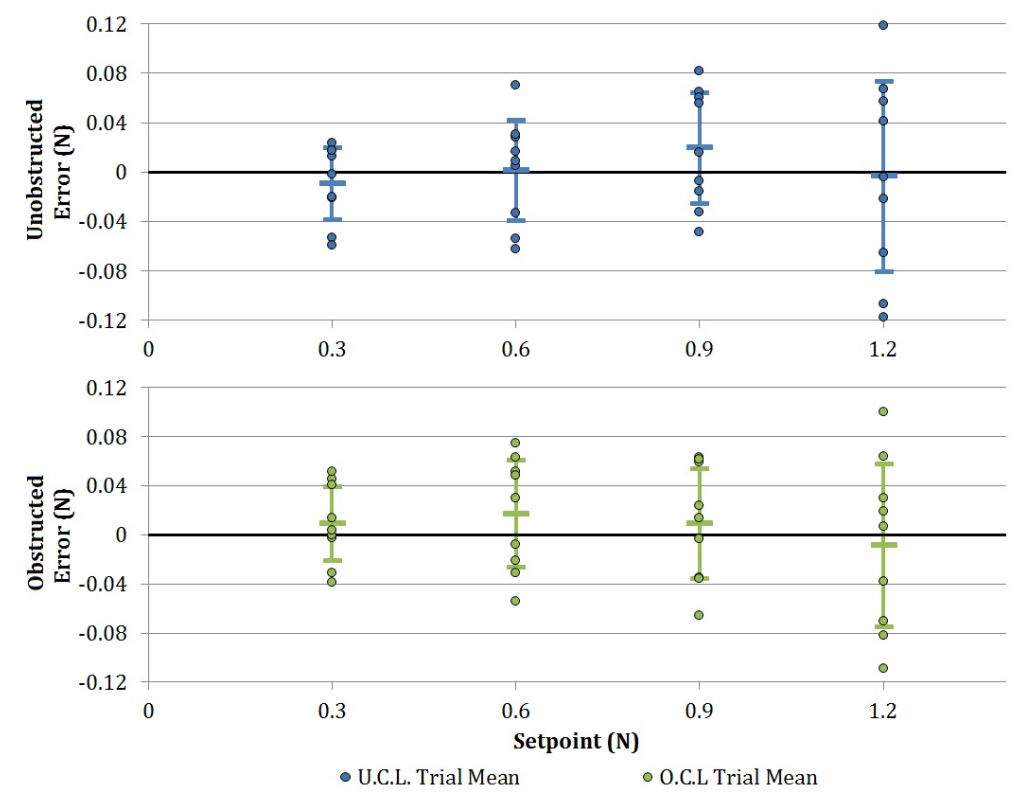

Figure 10: Closed loop radial force controller performance for 72 cutting trials, each lasting 60 seconds. Markers indicate mean force error for a given trial. Bars indicate standard deviation.

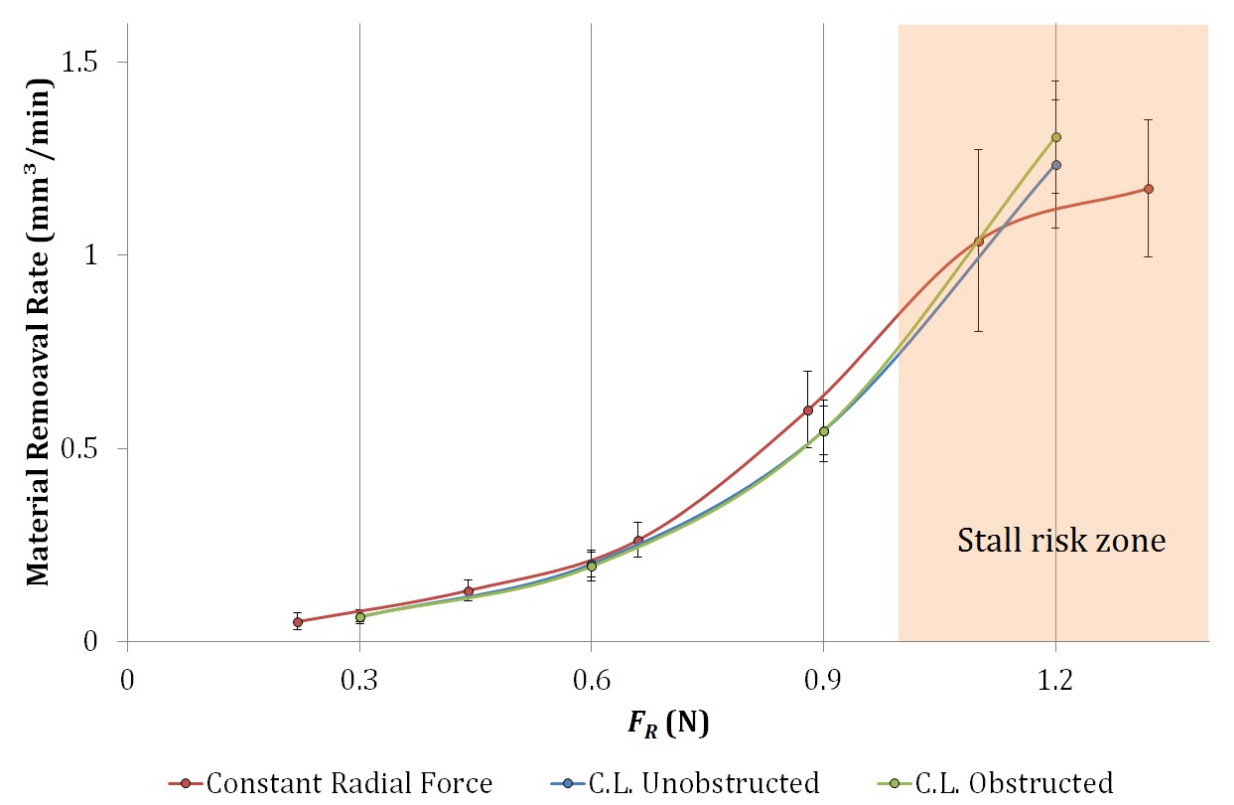

Figure 11: Material removal rates at known radial forces and using airborne sound features for closed loop radial force control.

The material removal rates achieved by the acoustic closed loop force controller (Figure 11) also align closely with the rates measured at known radial forces. This demonstrates that the closed loop control of spindle velocity using sound features provides an accurate way of controlling radial force during grinding. No substantial difference was observed between obstructed and unobstructed trials.

A stall risk zone of the air driven spindle was defined at radial forces greater than $1.0 \mathrm{~N}$. Contact force was found to oscillate significantly within the stall risk zone, often resulting in loss of power and void results. Despite this, the mean force of successful trials closely approaches the desired force, 
suggesting that regardless of the variance in frequency during calibration (attributed to oscillations in the offset mass setup), the calculated engagement coefficient closely describes the real system.

To evaluate the susceptibility of the proposed controller to tool wear, the cantilever test rig (Figure 9) was used to indent a workpiece at constant force using acoustic feedback force control for a long period of time. The contact force was set to $0.4 \mathrm{~N}$ and the bur was left in contact with the workpiece until a change in behaviour was observed. Contact was concentrated to a $1.6 \mathrm{~mm}$ portion of the tool to accelerate wear (ordinarily the entire length of the bur would be used). The results shown in Figure 12 indicate that force tracking remains effective far beyond the expected duration of in-situ tasks, with tool wear that far exceeds the threshold at which burs would ordinarily be replaced. After 30 minutes of continuous machining the controller was unable to track the frequency and started to overshoot widely, likely due to the reduced engagement coefficient.//

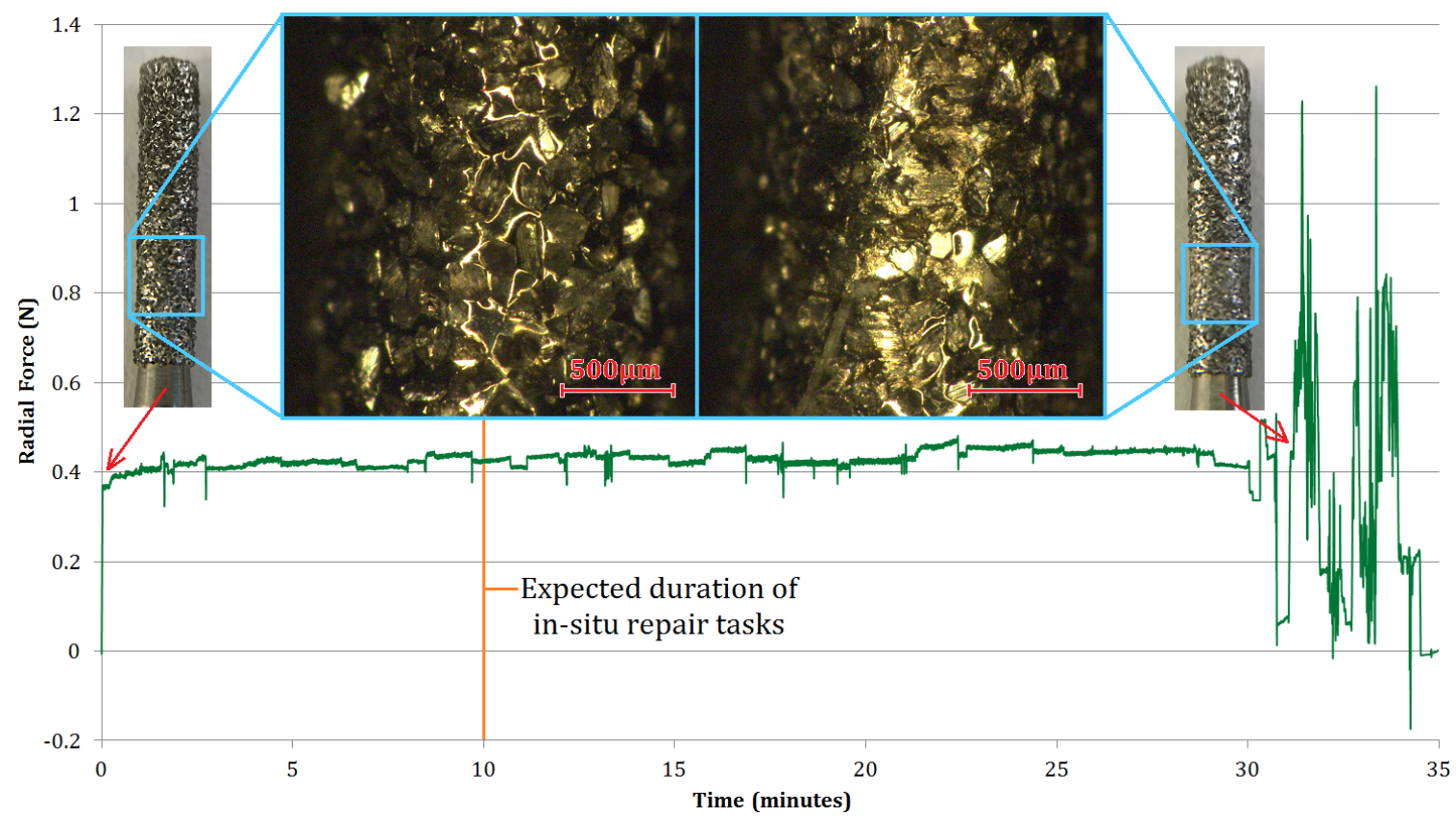

Figure 12: Tool wear experiment results show radial force tracking works effectively until control is lost at excessive wear levels.

Radial force was found to creep up (by approximately $10 \%$ over 30 minutes) during the longduration trials (Figure 12) This rise in contact force can be attributed to a lower engagement coefficient resulting from worn abrasive grits. While quantifying the influence of wear on the engagement coefficient is outside the scope of this work, it is important to understand the limitations of the proposed controller for applications that require prolonged in-situ machining with worn tools.

\subsubsection{Machining demonstration}

To validate the controller in a more realistic application, the grinding tool and L12-50-100-12-P DC geared linear actuator were attached to the end effector of a R12-5 5-degree-of-freedom robotic arm (ST Robotics; Figure 13). The robot arm was used to feed the tool back and forth, creating $90^{\circ}$ cuts on the edge of three different workpieces $(100 \mathrm{~mm} x 30 \mathrm{~mm} x 1.6 \mathrm{~mm})$ made from Nickel-Chromium-Cobalt alloy (Figure 14).

- A workpiece with a straight edge (Figure 14a)

- A workpiece with a concave indentation previously ground by hand (Figure 14b) 


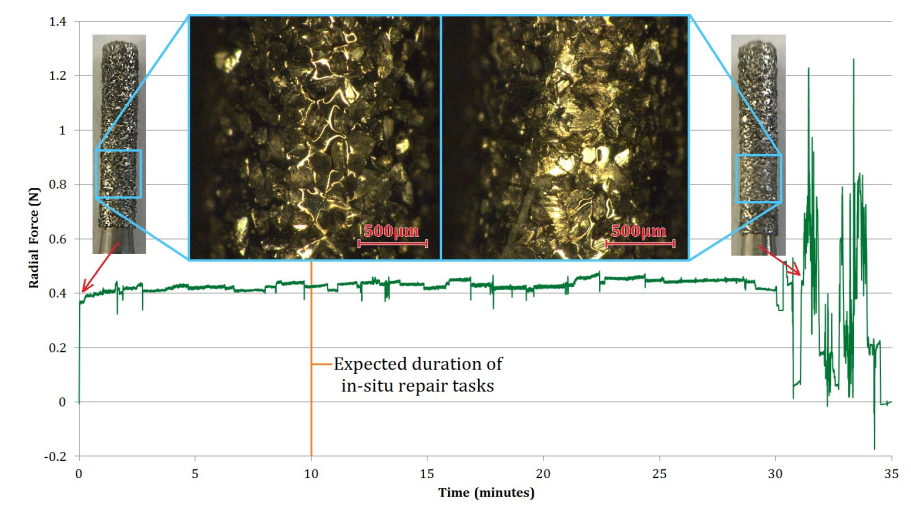

Figure 13: Demonstration setup for closed loop force control using sound features. 5-axis robot arm was used to control the feed speed $u_{f}$ along a cantilever beam and a linear actuator was used to control the tool radial force $F_{R}$.

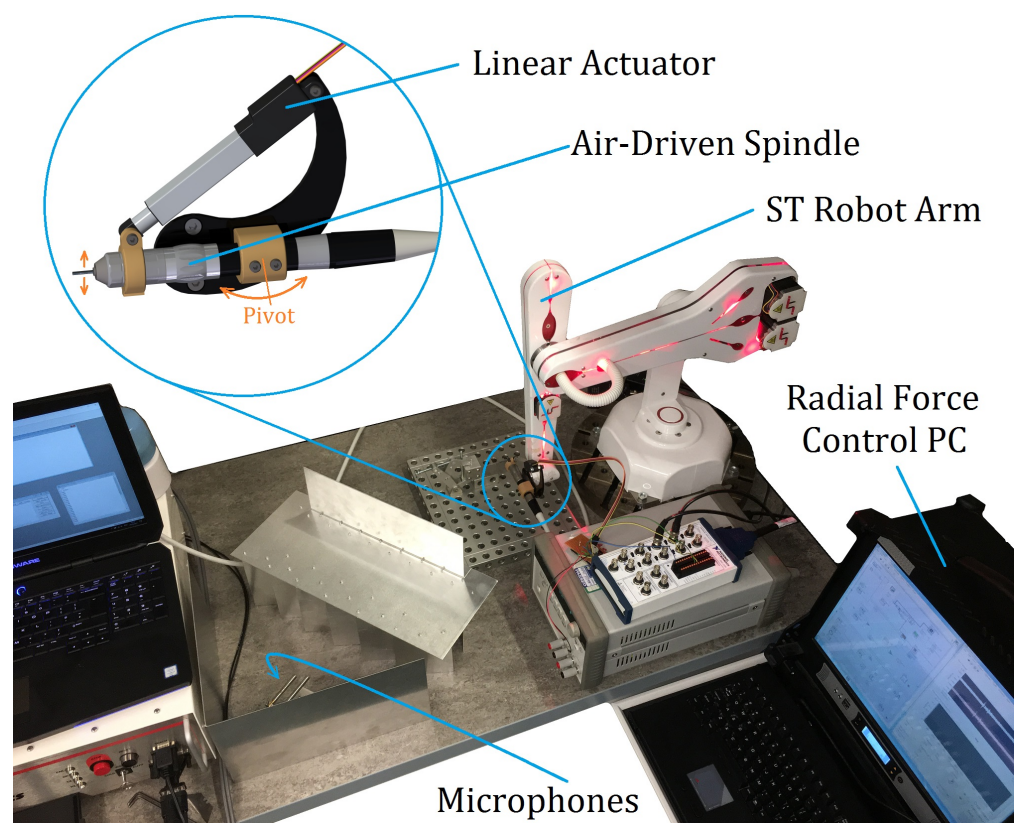

Figure 14: Demonstration results: A straight (a), concave (b) and convex (c) target workpiece geometry machined using a 5-DoF robot to feed the spindle and tool back and forth 50 times, and using audible sound features for closed loop force control. Red area shows removed material. Standard deviation of depth calculated from 10 samples taken at $1 \mathrm{~mm}$ intervals.

- A workpiece with a convex protrusion previously ground by hand (Figure 14c)

The irregular workpiece surfaces were used to test the ability of the sound force controller to adjust the radial motion axis (linear actuator in Figure 13) and follow an unknown path. It is important to note that the robot arm was not programmed to follow the different paths, but rather to maintain a constant radial force, hence a constant stock of removed material (depth of cut). Each of the tests consisted of fifty $10 \mathrm{~mm}$ passes at a feed rate of $u_{f}=1 \mathrm{~mm} / \mathrm{s}$ along the workpiece with a target force of $0.9 \mathrm{~N}$, resulting in an expected material removal rate of approximately $0.5 \mathrm{~mm}^{3} / \mathrm{min}$. Total material removed during the test is indicated by the red colour in Figure 14.

The robotic arm successfully removed material along the three different unknown surfaces using only the sound features to control radial force. An average material removal rate of $0.84 \mathrm{~mm}^{3} / \mathrm{min}$ 

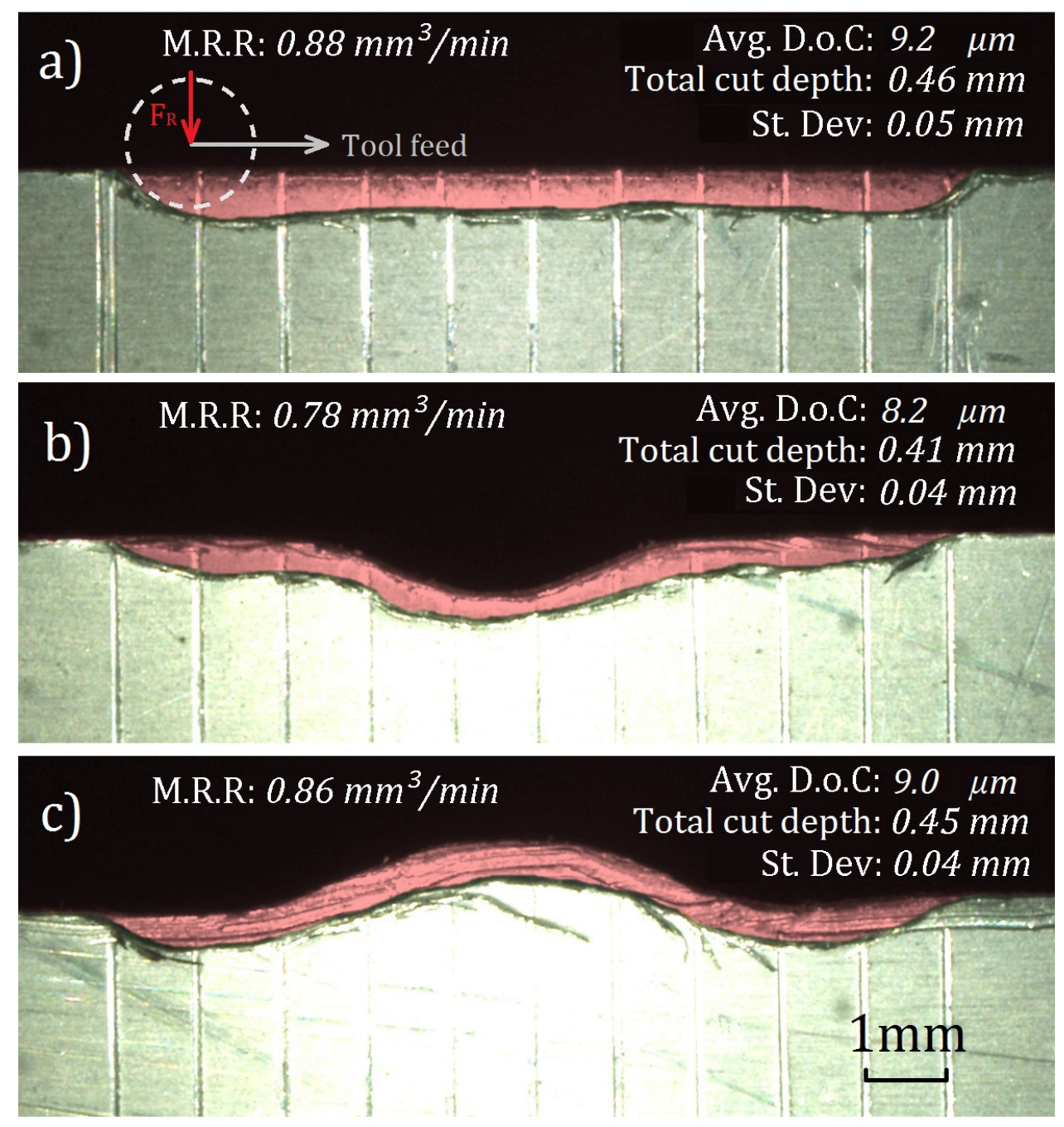

Figure 15: Radial force during first two passes of convex protrusion cut.

was measured with a standard deviation in depth of cut of $50 \mu \mathrm{m}$ or less (Figure 14). The fact that the material removal rate measured exceeds the rate obtained with the test rig (Figure 11) can be attributed to the smaller contact area (and hence higher pressure) between tool and workpiece due to a non-zero feed speed. Figure 15 shows filtered (lowpass, $100 \mathrm{~Hz}$ ) radial force data for the first two passes of the convex protrusion cut. The measurements show consistent force tracking, which was maintained throughout the majority of the trial.

The objective of this study is to demonstrate the application of a force controller using sound features with the intention that this methodology is applicable to other processes. In particular we are referring here to an in-situ geometrical correction of a component in an industrial asset, where the micro-aspects of surface quality (e.g. roughness) are less important. It is possible that future work could design tools optimised for specific applications such that, given a material, spindle speed tool type and machining force combination, machining quality and efficiency can be maximised (such as in the example of [26]). 


\section{Conclusions and future work}

This work shows that it is possible to use sound features to inform a controller and operate a machining instrument with closed loop force control. An adaptive proportional controller was designed to use inputs from both dominant frequencies and sound intensity to achieve a steady spindle velocity and hence a near constant radial force. The controller also uses changes in the intensity of sound to vary control parameters such as proportional gain, deadband width and filter settings to mitigate the risk of workpiece resonant frequencies affecting the control.

The closed loop control method described was able to closely match the calibration radial forces and replicate the material removal rate achieved using a constant grinding force test rig. The force controller was used to machine approx $0.44 \mathrm{~mm}$ deep features on thin cantilever beams with unknown edge profiles (concave, convex and straight) despite having no position control, thus demonstrating the ability to follow edges 'blind' using sound for force control. The susceptibility of the controller to small disturbances and variability in machining conditions means that the cuts produced had an uneven bottom with a standard deviation equal to $0.05 \mathrm{~mm}$ in depth of cut along the machining path. In future the airborne sound force controller could be used as part of a hybrid position-force controller to achieve optimal material removal rate as well as dimensional accuracy of the machining operation. Tests of the effect of tool wear on controller performance indicate that the method works appropriately for the useful life of the burs tested, despite losses in accuracy at high wear levels. In future work, the changes in performance could be further quantified and added to the controller for longer duration machining tasks.

The controller was demonstrated using a 5-axis robot arm with position encoders, but this method could be applied to operate non-conventional robots with limited position accuracy such as continuum and soft robots. Closed loop force control of such robots would allow for in-situ machining operations to be carried out in more inaccessible industrial components.

While the process described in this work is the high-speed grinding of metallic components, this methodology can be applied to other machining processes that produce audible sound, such as drilling, milling, polishing, sandblasting, laser ablation, etc., provided that the sound can be associated with one or more machining parameters.

Future work: The versatility and performance of this controller could be improved by continuously processing the sound signals, such as by using a field programmable gate array (FPGA) for intensity and power spectral density calculations. This would allow the control loop to operate with lower latency and therefore at higher frequencies. This would in turn allow for more complex control structures such as PID to tune the response of the controller to perturbations in machining forces.

The controller could also be made more robust by introducing a cascade controller with an inner velocity control loop for the actuator, ensuring more predictable response and better control authority. Additionally, extra microphones could be used for noise cancellation to allow such a force control strategy to be used in noisy industrial settings.

Finally, it is possible to optimise the resulting surface characteristics for particular workpiece and tool combinations. This would achieve improved surface quality, material removal rate and tool life while employing air spindle speed-based force control. This will require modelling the behaviour of the tool at different stages of tool wear. Such work could result in quality consistent with the high standards associated with the aerospace and energy industries.

\section{References}

[1] X. Dong, D. Axinte, D. Palmer, S. Cobos, M. Raffles, A. Rabani, and J. Kell. Development of a slender continuum robotic system for on-wing inspection/repair of gas turbine engines. Robotics and Computer-Integrated Manufacturing, 44:218-229, 2017. 
[2] X. Dong, M. Raffles, S. C. Guzman, D. Axinte, and J. Kell. Design and analysis of a family of snake arm robots connected by compliant joints. Mechanism and Machine Theory, 77:73-91, 2014 .

[3] K. Xu and N. Simaan. An investigation of the intrinsic force sensing capabilities of continuum robots. IEEE Transactions on Robotics, 24(3):576-587, 2008.

[4] A. Bajo and N. Simaan. Hybrid motion/force control of multi-backbone continuum robots. International Journal of Robotics Research, 35(4):422-434, 2016.

[5] R. E. Goldman, A. Bajo, and N. Simaan. Compliant motion control for multisegment continuum robots with actuation force sensing. IEEE Transactions on Robotics, 30(4):890-902, 2014.

[6] S. J. Phee, S. C. Low, P. Dario, and A. Menciassi. Tendon sheath analysis for estimation of distal end force and elongation for sensorless distal end. Robotica, 28:1073-1082, 2010.

[7] C. Liu, G. F. Wang, X. D. Qin, and L. Zhang. The online monitoring of surface quality based on time-frequency spectrum analysis of acoustic emission. Functional Manufacturing and Mechanical Dynamics Ii, 141:564-568, 2012.

[8] T. W. Liao, F. M. Tang, J. Qu, and P. J. Blau. Grinding wheel condition monitoring with boosted minimum distance classifiers. Mechanical Systems and Signal Processing, 22(1):217-232, 2008.

[9] A. Rabani, I. Marinescu, and D. Axinte. Acoustic emission energy transfer rate: A method for monitoring abrasive waterjet milling. International Journal of Machine Tools $\&$ Manufacture, 61:80-89, 2012.

[10] Z. J. Lv, Q. Xiang, and J. Z. Pang. An artificial immune algorithm based intelligent monitoring system in grinding process. Manufacturing Process Technology, Pts 1-5, 189-193:2759-2763, 2011.

[11] T. Blum and I. Inasaki. A study on acoustic-emission from the orthogonal cutting process. Journal of Engineering for Industry-Transactions of the Asme, 112(3):203-211, 1990.

[12] W. Konig, Y. Altintas, and F. Memis. Direct adaptive-control of plunge grinding process using acoustic-emission (ae) sensor. International Journal of Machine Tools 8 Manufacture, 35(10):1445-1457, 1995.

[13] R. H. Sturges. Monitoring milling processes through ae and tool part geometry. Journal of Engineering for Industry-Transactions of the Asme, 114(1):8-14, 1992.

[14] Y. G. Zeng and E. Forssberg. Application of vibration signal measurement for monitoring grinding parameters. Mechanical Systems and Signal Processing, 8(6):703-713, 1994.

[15] I. Buj-Corral, J. Alvarez-Florez, and A. Dominguez-Fernandez. Acoustic emission analysis for the detection of appropriate cutting opperations in honing processes. Mechanical Systems and Signal Processing, 99(1):873-885, 2018.

[16] E. M. Rubio and R. Teti. Cutting parameters analysis for the development of a milling process monitoring system based on audible energy sound. Journal of Intelligent Manufacturing, 20(1):43$54,2009$.

[17] P. Iskra and C. Tanaka. A comparison of selected acoustic signal analysis techniques to evaluate wood surface roughness produced during routing. Wood Science and Technology, 40(3):247-259, 2006. 
[18] T. Thaler, P. Potocnik, I. Bric, and E. Govekar. Chatter detection in band sawing based on discriminant analysis of sound features. Applied Acoustics, 77:114-121, 2014.

[19] L. Robben, S. Rahman, J. C. Buhl, B. Denkena, and B. Konopatzki. Airborne sound emission as a process monitoring tool in the cut-off grinding of concrete. Applied Acoustics, 71(1):52-60, 2010 .

[20] M. A. Khan, K. Basit, S. Z. Khan, K. A. Khan, and A. G. Starr. Experimental assessment of multiple contact wear using airborne noise under dry and lubricated conditions. Proceedings of the Institution of Mechanical Engineers Part J-Journal of Engineering Tribology, 231(12):1503-1516, 2017.

[21] Ioan D. Marinescu. Handbook of machining with grinding wheels. CRC Press, Taylor \& Francis Group, CRC Press is an imprint of the Taylor \& Francis Group, Boca Raton, second edition. edition, 2016.

[22] Bill Gunston. The development of jet and turbine aero engines. Patrick Stephens, Sparkford, Somerset, 2nd edition, 1997.

[23] J. E. Dyson and B. W. Darvell. Torque, power and efficiency characterization of dental air turbine handpieces. Journal of Dentistry, 27(8):573-586, 1999.

[24] U. S. P. Durgumahanti, V. Singh, and P. V. Rao. A new model for grinding force prediction and analysis. International Journal of Machine Tools \& Manufacture, 50:231-240, 2010.

[25] Finn Jacobsen and Peter Moller Juhl. Fundamentals of general linear acoustics. John Wiley \& Sons Inc., Chichester, West Sussex, United Kingdom, 2013.

[26] A. V. Gopal and P. V. Rao. The optimisation of the grinding of silicon carbide with diamond wheels using genetic algorithms. International Journal of Advanced Manufacturing Technology, 22(7):475-480, 2003. 